\title{
The Efficacy of Medial Patellofemoral Ligament Reconstruction Combined with Tibial Tuberosity Transfer in the Treatment of Patellofemoral Instability
}

\author{
Tarek Boutefnouchet, MBChB, MRCS, PGCME ${ }^{1,2}$, Christopher Downham, MBChB, MRCS ${ }^{1}$, \\ James Bassett, MBChB, MRCS ${ }^{1}$, Peter Thompson, MBChB, FRCS (TR\&Orth) ${ }^{1}$, and \\ Andrew Sprowson, MBChB, PhD, FRCS (TR\&Orth) ${ }^{1,2}$ \\ ${ }^{1}$ Department of Trauma and Orthopaedic, University Hospital Coventry and Warwickshire, Coventry; ${ }^{2}$ Warwick Medical School, The University of Warwick, \\ Coventry, United Kingdom
}

\begin{abstract}
A systematic review of the literature was undertaken to evaluate the efficacy of medial patellofemoral ligament (MPFL) reconstruction combined with tibial tuberosity transfer (TTT) in the treatment of patellofemoral instability. Using PRISMA (Preferred Reporting Items for Systematic Reviews and Meta-Analyses) guidelines, a systematic search was carried out to identify and review the published literature pertinent to MFPL reconstruction combined with TTT. Relevant studies were critically appraised with narrative data synthesis. Studies that met the eligibility criteria were suitable for appraisal and consisted of case series and therapeutic series (levels IV \& III). All studies had inherent variations in outcomes reporting and limited follow-up. Combined treatment offers restoration of normal anatomy, thus adding clinical value to the currently recommended anatomic approach to MPFL reconstruction. Nevertheless, the current body of evidence does not determine the threshold at which patellofemoral axis requires the need for adjunctive distal realignment as opposed to MPFL reconstruction alone. This review highlighted numerous recurring limitations in the conduct and presentation of the studies, which inadvertently mitigated the interpretation of their results. Future priority should be awarded to larger randomised controlled trials utilising validated patient reported outcome measures.
\end{abstract}

Keywords: Patellofemoral instability, Patellar dislocation, Medial patellofemoral ligament reconstruction, Tibial tuberosity osteotomy

\section{Introduction}

Patellar instability is a painful and debilitating condition that usually affects young active patients. Acute traumatic primary patellar dislocation has a reported annual incidence of 77.4 per 100,000 persons $^{1)}$. Recurrent patellofemoral dislocation and as-

Received June 3, 2015; Revised (1st) August 25, 2015;

(2nd) September 27, 2015; Accepted October 19, 2015

Correspondence to: Tarek Boutefnouchet, MBChB, MRCS, PGCME

Department of Trauma and Orthopaedic, University Hospital Coventry and Warwickshire, Clifford bridge road, Walsgrave, Coventry, CV2 2DX, United Kingdom

Tel: +44-0-7809677302, Fax: +44-0-2476965098

E-mail: tboutefnouchet@hotmail.com

This is an Open Access article distributed under the terms of the Creative Commons Attribution Non-Commercial License (http://creativecommons.org/licenses/by-nc/4.0/) which permits unrestricted non-commercial use, distribution, and reproduction in any medium, provided the original work is properly cited. sociated lesions such as trochlear dysplasia constitute major risk factors for the development of premature focal osteoarthritis ${ }^{2}$. Stability of the patellafemoral joint is maintained by a number of structures and the medial patellofemoral ligament (MPFL) acts as the primary stabiliser during the initial $20^{\circ}$ to $30^{\circ}$ of knee flexion $^{3)}$. The MPFL has a reported rupture rate of $90 \%-100 \%$ following lateral patellar dislocation ${ }^{1,3,4)}$. A relatively high rate of recurrent instability after dislocation is reported between $40 \%$ and $60 \%{ }^{3)}$. Focusing surgical management on the reconstruction of the MPFL is therefore an increasingly attractive option for many surgeons. Cadaveric studies, which performed transection of the MPFL, resulted in abnormal lateral tracking with markedly increased lateral translation and tilt as well as significantly increased lateral patellar facet joint reaction forces ${ }^{5}$. The same group in a different study reported the risk of over constraints on the patellofemoral joint with even a small variation in the anatomical reconstruction of the $\mathrm{MPFL}^{6}$. . Similarly, Philippot et 
al. ${ }^{7)}$ demonstrated that increased tension of MPFL reconstruction results in abnormal and potentially detrimental patellofemoral kinematics. This is supported in other studies which showed the risk of increased medial patellar facet contact pressure following MPFL reconstruction ${ }^{8)}$. Other recent studies have demonstrated that MPFL reconstruction may not be as forgiving as originally predicted. Camp et al. ${ }^{9)}$ reported a failure rate of $28 \%$ with recurrent lateral patellar dislocation after isolated MPFL reconstruction at a mean follow-up of 4 years. Other potential short-term complications include reduced range of movement, due to graft over-tension or shortening during flexion ${ }^{10)}$. Such issues raise the question of whether MPFL reconstruction alone is the correct option for managing patellofemoral instability.

MPFL reconstruction has also been combined with tibial tuberosity medialisation (TTM) and tibial tuberosity distalisation aiming to correct patellofemoral alignment and place the patella in a more biomechanically advantageous position, thus restoring stability ${ }^{11,12)}$. Tuberosity medialisation has been shown to decrease lateral patellar facet joint pressure by $15 \%$ at $60^{\circ}$ to $80^{\circ}$ of knee flexion, without overloading the medial facet articulation $^{13)}$. Nevertheless, a lack of consensus prevails on the threshold and the exact indications for bony realignment in patellofemoral instability ${ }^{11,14,15)}$. Caplan et al. ${ }^{16)}$ demonstrated a lack of difference in tibial tuberosity trochlear groove (TT-TG) distance between stable and unstable patellofemoral joints in a cohort of patients with unilateral instability. In addition, the long-term impact on soft tissue balancing and chondral surface wear associated with such procedures remains highly underreported. A recent in vitro study showed altered tibiofemoral kinematics following tibial tuberosity medialisation with an ensuing increase in tibial external rotation, posterior translation and varus orientation ${ }^{17)}$.

It is well recognised that patellofemoral instability is a multimodal condition associated with osteochondral abnormality in the form of trochlear dysplasia, patellofemoral malalignment with: increased height, increased lateral offset and patellotrochlear mismatch, as well as disruption of medial soft tissue restraints ${ }^{11,12,18)}$. Therefore, patellar instability cannot be defined as a single entity condition but rather as an abnormality resulting from a constellation of conditions. The terms 'objective patellar instability' and 'episodic patellar dislocation' have been coined ${ }^{18,19)}$ and this terminology not only reflects the broad spectrum nature of the condition but also gives rise to a better understanding in the variation of indications for the different surgical options. Many questions remain unanswered: on whether a wide fan shaped ligament replaced by a tighter tubular shaped graft is the principal solution to patellar instability ${ }^{20,21)}$; and whether a tighter medial constraint following MPFL surgery is a desired side effect to compensate for patellofemoral malalignment conditions ${ }^{21)}$ ? In addition, there is a clear lack of consensus on the indications for adding distal bony realignment procedure to MPFL reconstruction. Given the multifactorial properties of patellar instability, it seems instinctive to consider the solution to be equally multifactorial. This systematic review explored the growing body of research reporting outcomes of MPFL reconstruction in order to appraise the evidence for combining tibial tuberosity transfer (TTT) procedures. Our aim was to answer specific questions on: 1) whether combined treatment confers better outcomes for patellofemoral instability, and 2) whether there is an identifiable subset of patients in whom combined treatment is indicated.

\section{Methods}

A systematic review of the literature was performed according to the methods described in the PRISMA (Preferred Reporting Items for Systematic Reviews and Meta-Analyses) statement, and summarised on the flowchart in Fig. ${ }^{22)}$. Search database used were: MEDLINE, EMBASE, CINAHL (Cumulative Index to Nursing and Allied Health Literature), and the Cochrane CENTRAL (Central Register of Controlled Trials). Search dates intervals included were from their year of inception to June 2014. The searches were limited to humans and English language. PubMed was used as the primary source and search engine for MEDLINE and $\mathrm{MeSH}$ were used, Table 1 summarises the search terms and strategies utilised.

\section{Eligibility Criteria}

Studies included were original articles pertinent to our research questions. The criteria for eligibility were as shown in the study parameters below with studies using any of the outcomes included within the review:

1) Participants included were skeletally mature patients with reported recurrent patellar dislocation or subluxation as well as objective patellar instability, undergoing primary surgical treatment.

2) Interventions evaluated consisted of MPFL reconstruction using autogenous tendon grafts of any type; in combination with procedures aimed at restoring patellofemoral alignment which pragmatically consisted of TTT including: elevation, medialisation, distalisation or a combination of these techniques.

3) Comparison intervention consisted of isolated soft tissue procedure intervention as above aimed at reconstructing the 


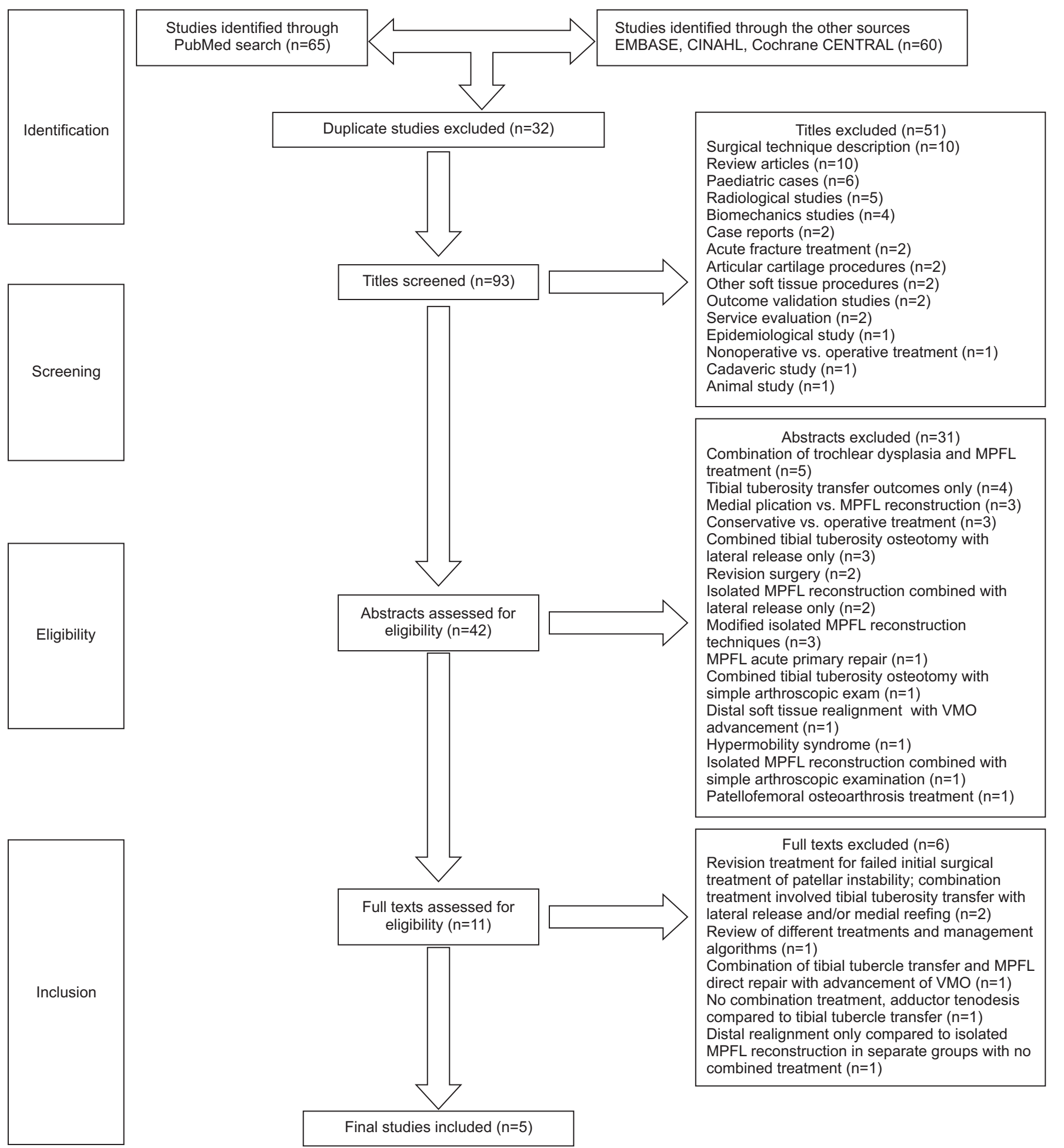

Fig. 1. Flow diagram of the systematic literature search on combined medial patellofemoral ligament (MPFL) reconstruction and tibial tuberosity transfer. CINAHL: Cumulative Index to Nursing and Allied Health Literature, CENTRAL: Central Register of Controlled Trials, VMO: vastus medialis obliquus. (c) Reproduced with permission of the PRISMA IPD Group, which encourages sharing and reuse for non-commercial purposes. 
Table 1. Database, Search Terms, and Search Strategy Used in the Systematic Review of Current Evidence

\begin{tabular}{|c|c|}
\hline & Systematic review \\
\hline Database & PubMed, EMBASE, CINHAL, Cochrane CENTRAL \\
\hline Search terms & $\begin{array}{l}\text { Patella, patellar, instability, dislocation, subluxation, luxation, maltracking, hyperlaxity, MPFL, reconstruction, surgery, } \\
\text { procedure, tibial tuberosity, transfer, Fulkerson, Elmslie-Trillat, osteotomy, procedure }\end{array}$ \\
\hline Search strategy & 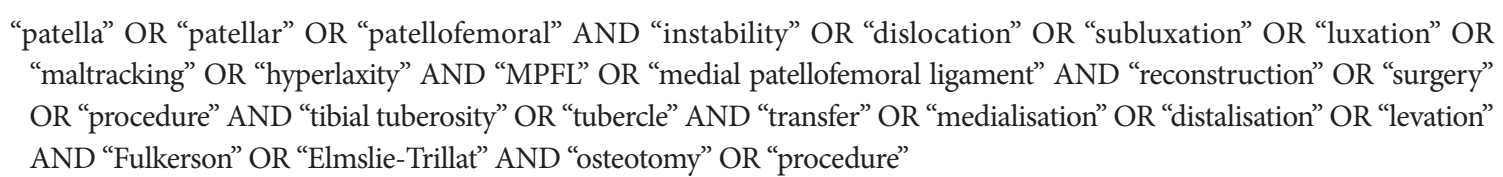 \\
\hline
\end{tabular}

CINAHL: Cumulative Index to Nursing and Allied Health Literature, CENTRAL: Central Register of Controlled Trials, MPFL: medial patellofemoral ligament.

MPFL.

4) Outcomes included in the review were recurrence of dislocation or subluxation and functional results of treatment as surrogate marker of measurement of patellofemoral instability symptoms.

5) Primary outcome measure was the incidence of patellar redislocation for studies which reported on treatments carried out for recurrent patellar dislocations.

6) Secondary outcome measure evaluated was the Kujala score, a validated score for patellofemoral symptoms and function. This scoring system is self-administered and weighted, and comprises 13 elements ranging from 0 to 100 , higher scores indicating better performance ${ }^{9,23)}$. The elements related to patellar stability are valid and reliable for the assessment of such patellofemoral conditions. Studies which fulfilled all the primary inclusion criteria but utilised different outcome measures were included in the review.

\section{Exclusion Criteria}

Studies excluded from this review were: case reports, technical reports, review articles and abstracts only or conference proceedings publications. Studies looking at post-arthroplasty patellofemoral instability, post-trochleoplasty instability, revision surgery, feasibility studies, studies on cost effectiveness, cadaveric studies, studies analysing the biomechanics of patellofemoral malalignment were excluded. Studies which included combination of simple arthroscopic examination, arthroscopic chondroplasty, and/or microfractures were not considered as relevant patellofemoral combinations procedures and were therefore excluded.

\section{Review Process and Analyses}

Articles which met the inclusion criteria were systematically assessed for the relevance of their content (Fig. 1). Initially titles were screened for primary inclusion and exclusion. All the abstracts obtained were further assessed for eligibility. The full texts of articles which met the relevance and inclusion criteria were obtained and reviewed, paying particular attention to relevance to our research questions. Only studies with separate outcome measures specific to combination of MPFL reconstruction with TTT were included in the final review (Fig. 1). Access to full text articles was obtained from Athens (Eduserv, Bath, UK), Warwick university library, and Google Scholar. A rigorous systematic search was performed using the criteria outlined above. References were transferred into EndNote (Thomson Reuters, Philadelphia, PA, USA) and duplicates were discarded. Firstly titles and abstracts were reviewed for relevance according to the research question. The remaining studies were analysed in their entirety. References of full texts were also reviewed to identify any other potentially relevant studies. The acquisition of articles is summarised in the flow diagram (Fig. 1). The final studies were reviewed according to study design, analysis and interpretation as well as validity of results. Eligible studies were critically appraised with narrative data synthesis relevant to the study designs. All relevant studies were critically appraised by two independent reviewers (TB and $\mathrm{CD}$ ). Where there was discrepancy, an agreement was reached by consensus. The critical appraisal followed a systematic approach guided by the consolidated standards of reporting trials for randomised studies ${ }^{24}$, and the validated methodological index for non-randomised studies ${ }^{25}$. A comprehensive critical appraisal checklist was created and used alongside the final papers included in the review (Table 2).

\section{Results}

\section{Results of Systematic Search}

A total of 125 studies were generated and 93 titles initially screened after removal of 32 duplicates. A further search expanded through the cited references did not yield additional eligible studies. Following initial screening and application of the inclusion criteria 51 titles and 31 abstracts were excluded from the 


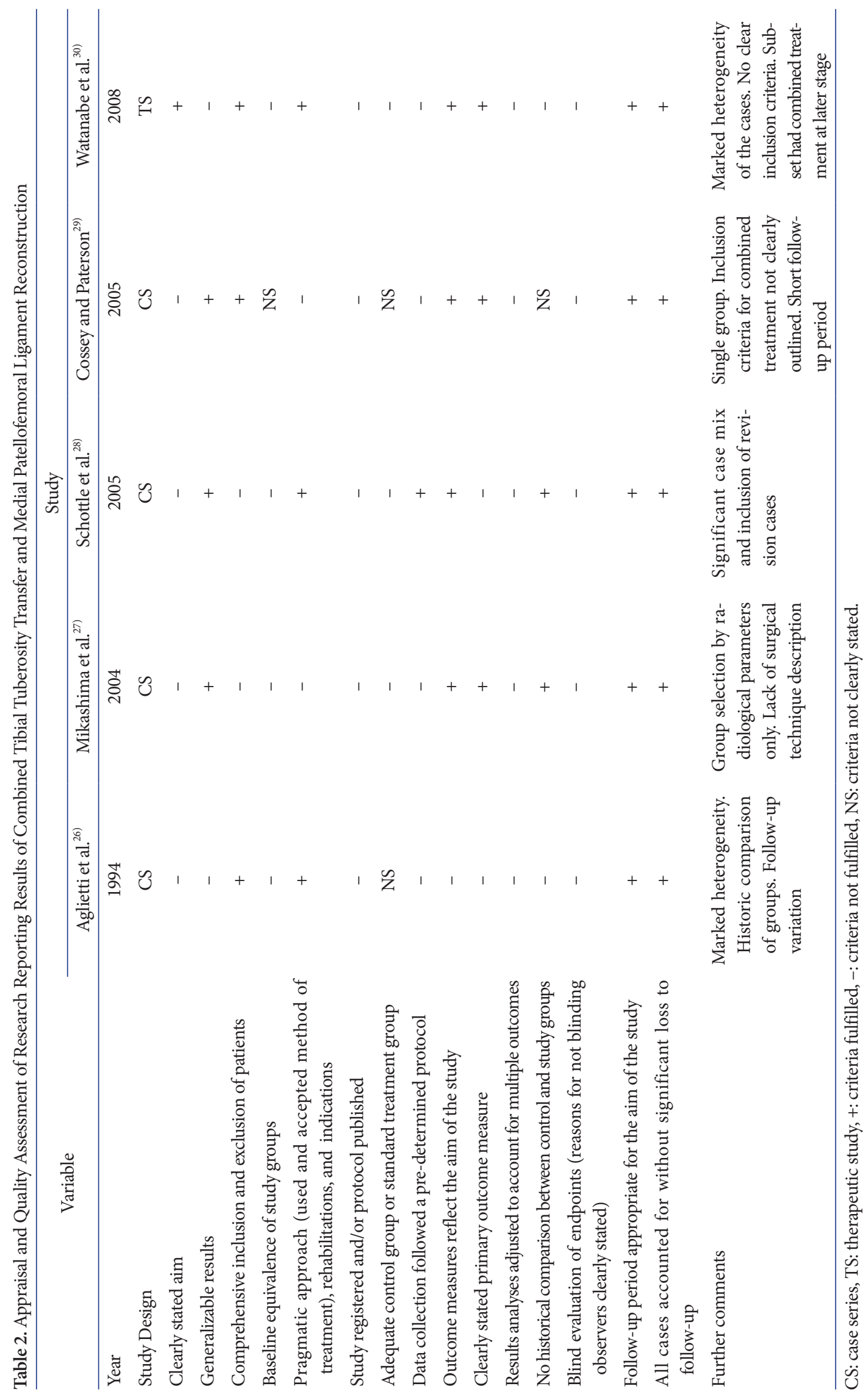


Table 3. Summary of Studies Included in the Final Analysis

\begin{tabular}{|c|c|c|c|c|c|c|c|}
\hline Study & Year & Case & Intervention & Subgroups & $\begin{array}{l}\text { Average F/U } \\
\text { (range) }\end{array}$ & Outcomes (range) & $\begin{array}{c}\text { Recurrent } \\
\text { dislocations }\end{array}$ \\
\hline \multirow[t]{4}{*}{ Aglietti et al. ${ }^{26)}$} & \multirow[t]{4}{*}{1994} & \multirow[t]{4}{*}{69} & LR & 20 & 8 yr $(5-10)$ & \multirow{4}{*}{$\begin{array}{l}\text { Normal tracking: } 43 \%-69 \% \\
\text { (-) apprehension sign: } 68 \%-86 \%\end{array}$} & \multirow[t]{4}{*}{ NR } \\
\hline & & & MPFL recon & 14 & 8 yr $(2-14)$ & & \\
\hline & & & $\mathrm{TTT}+\mathrm{LR}$ & 16 & 6 yr $(2.5-8)$ & & \\
\hline & & & TTT+LR+MPFL recon & 19 & $4 \mathrm{yr}(2-9)$ & & \\
\hline \multirow[t]{2}{*}{ Mikashima et al. ${ }^{27)}$} & \multirow[t]{2}{*}{2004} & \multirow[t]{2}{*}{40} & TTT & 20 & \multirow[t]{2}{*}{$41.0 \mathrm{mo}(28-52)$} & $6 / 20(+)$ apprehension, $3.0 \mathrm{~m}$ to full $\mathrm{ROM}$, & \multirow[t]{2}{*}{ NR } \\
\hline & & & TTT+MPFL recon & 20 & & $\begin{array}{l}\text { Kujala } 79.6(56-100) \\
0 / 20(+) \text { apprehension, } 2.1 \mathrm{~m} \text { to full ROM, } \\
\text { Kujala } 89.0(63-100)\end{array}$ & \\
\hline \multirow[t]{2}{*}{ Schottle et al. ${ }^{28)}$} & \multirow[t]{2}{*}{2005} & \multirow[t]{2}{*}{15} & MPFL recon & 7 & \multirow[t]{2}{*}{$47.0 \mathrm{mo}(24-70)$} & 13/15 good to excellent Kujala 85.7 & \multirow[t]{2}{*}{1} \\
\hline & & & MPFL recon+ TTT & 8 & & & \\
\hline $\begin{array}{l}\text { Cossey and } \\
\text { Paterson }^{29)}\end{array}$ & 2005 & 21 & $\mathrm{MPFL}$ recon $+\mathrm{TTT}+\mathrm{LR}$ & NA & $23.0 \mathrm{mo}(11-37)$ & $\begin{array}{l}\text { Lysholm } 95.6 \\
\text { Turba: good-excellent }\end{array}$ & Nil \\
\hline \multirow[t]{2}{*}{ Watanabe et al. ${ }^{30)}$} & \multirow[t]{2}{*}{2008} & \multirow[t]{2}{*}{42} & MPFL & 29 & \multirow[t]{2}{*}{$4.3 \mathrm{yr}(1.5-8.1)$} & Lysholm 92.4 & \multirow[t]{2}{*}{ NR } \\
\hline & & & $\mathrm{TTT}+\mathrm{MPFL}$ recon & 13 & & & \\
\hline
\end{tabular}

F/U: follow-up, LR: lateral release, NR: not reported, MPFL: medial patellofemoral ligament, recon: reconstruction, TTT: tibial tuberosity transfer, ROM: range of motion, NA: not available.

review (systematic review flowchart). Overall 11 studies met the eligibility criteria and full text review was carried out. A further 6 articles were excluded due to absence of combination treatment involving MPFL reconstruction and tibial tubercle transfer in all patients or a distinct patients sub-group within these studies. The following section examines the five articles included. The critical appraisal process for each study is outlined in Table 2 . The summarised results of these studies are presented in Table 3 , and Table 4 outlines the results specific to subgroups of combined TTT and MPFL reconstruction. All the reviewed final studies consisted of small retrospective series with inadequately defined selection criteria introducing selection bias. These studies utilised a vast plethora of surgical treatment techniques and the preoperative parameters defining patellofemoral instability were of great variation. This has led to difficulties in comparing outcomes of individual treatment options. Due to this marked heterogeneity it was not possible to combine the results for meta-analysis of the studies presented.

\section{Narrative Data Synthesis}

Aglietti et al. ${ }^{26)}$ reported the results of four different treatment approaches employed in the management of patellar instability among 67 patients ( 69 knees). All cases had two or more episodes of patellar dislocation and an analysis was carried out on pre and postoperative clinical and radiological evaluation. A subgroup of patients (19 knees) received a combination treatment which consisted of TTT, lateral release and MPFL reconstruction. In all cases treated with combined treatment, patellofemoral alignment was restored, $68 \%$ and $32 \%$ reported excellent and good stability outcomes respectively. There were similar overall clinical results in MPFL reconstruction and combined TTT and MPFL reconstruction. The combined treatment group had a higher number of pre-surgical dislocation, earlier onset dislocations and longer intervals to treatment.

Mikashima et al. ${ }^{27)}$ reported the results of a retrospective analysis of two randomly selected therapeutic groups. All patients presented with recurrent patellar dislocations and were subdivided into four subgroups depending on patellar tilt and tibial tuberosity offset. The 1st group consisted of 20 knees treated with TTT (Elmslie-Trillat procedure) alone. The 2 nd group consisted of 20 knees treated with MPFL reconstruction combined with a similar TTT procedure. There was no significant difference in muscle strength assessment, while the combined group had a quicker regain of full range of motion, mean 3.0 vs. 2.1 months. The group treated with TTT alone reported a mean postoperative Kujala score of 79.6 (range, 56 to 100), compared to 89.0 (range, 63 to 100 ) in the group which received combined treatment.

Schottle et al. ${ }^{28)}$ published a case series in which they reported the results of 12 patients ( 15 knees). Patients selected in this study had either two or more episodes of patellar dislocations or one dislocation followed by persistent positive patellar apprehension sign. Within the cases selected a subset of eight knees had 


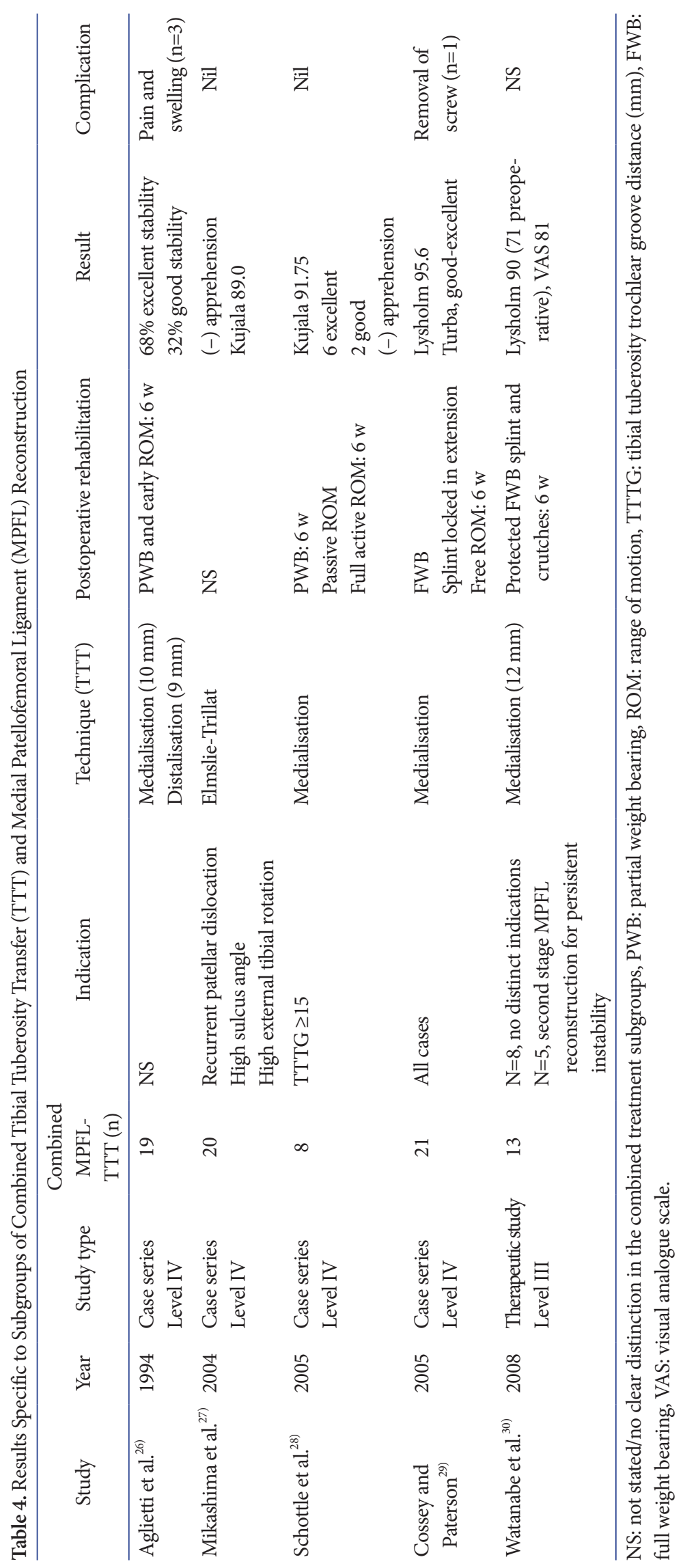


a combination of MPFL reconstruction and TTT in the form of medialisation indicated by a TT-TG distance of more than 15 $\mathrm{mm}$. The subgroup of patients with combined TTT and MPFL reconstruction reported a mean Kujala score of 91.75 (63.60, preoperatively) and six knees had excellent and two good results on subjective clinical evaluation. In this subgroup there was no recurrent patellar instability and the postoperative apprehension sign was negative in all cases.

Cossey and Paterson ${ }^{29)}$ reported the results of patellar instability treatment in 19 patients ( 21 knees). All cases were treated with a combination of lateral release, MPFL reconstruction using medial retinaculum graft, and TTT. The postoperative outcomes at the last follow-up showed good to excellent results in all cases on Turba score, the mean Lysholm score was 95.6. They reported that all cases had restoration of their functional status on Tegner scores and no incidence of recurrent patellar instability.

Watanabe et al. ${ }^{30)}$ reported the results comparing the clinical outcomes of MPFL reconstruction with those of MPFL reconstruction combined with TTT. This study comprised a total of 40 patients (42 knees) treated with hamstring autograft MPFL reconstruction with or without TTT in the form of medialisation. Lysholm score improved from 72 to 90 in the combined MPFLTTT group. The postoperative subjective evaluation using a visual analogue score demonstrated a significantly higher score in the MPFL only group. No incidence of recurrent dislocation was reported in either group.

\section{Critical Appraisal}

All the studies included in the systematic review suffered from combined and repeated methodological deficiencies (Table 2). There was a lack of clear statement indicating the primary objective of the study and whether a comparison of the efficacy of treatment methods was the primary endpoint. Exclusion of patients who received treatment but did not have their outcomes reported were not clearly outlined. These methodological limitations also included a lack of prospective calculation of sample size, power and level of significance; and no evidence of study registration or conduct according to a predefined and registered study protocols. Due to the lack of intransigent evidence supporting one or more treatment options there was no scope for adequate standard control comparison groups. In addition, there was no evidence of blind assessment of objective study endpoints with lack of statements justifying the absence of blinding. The studies by Mikashima et al. ${ }^{27)}$ and Schottle et al. ${ }^{28)}$. used the Kujala score. Although this score is validated, and widely used with satisfactory test-retest reliability, it remains a limited patient re- ported outcome measure since the last two questions are clinical examination findings ${ }^{23,31)}$. Similarly, Paxton et al. ${ }^{31)}$ showed that the Lysholm score is a more sensitive scale, in particular for differentiating between patients with and without recurrent subluxations or dislocations. It also appears that on the whole the duration of follow-up in all the studies was dictated by the availability of postoperative outcome data rather than a reflection of the impact of treatment or according to a published study protocol.

\section{Discussion}

This systematic review of the literature highlights the paucity of high quality controlled studies investigating combined MPFL reconstruction with tibial tuberosity realignment for patellar instability. Such literature remains poor with heterogeneous cases often including objective patellar instability alone alongside the different entity of patellofemoral malalignment with one or more anatomical abnormality. Patellofemoral instability is a condition characterised by abnormal movements associated with recurrent subluxation and/or dislocation of the patella ${ }^{10)}$. The last two decades have witnessed a significant expansion in the understanding of the pathoanatomy of patellar instability, and this has translated into the development of numerous surgical techniques. The performance of additional bony realignment procedure remains controversial, with lack of evidence on the cut-off TT-TG value indicating the need for TTT. Matthews and Schranz ${ }^{32)}$ in a single group case series reported good results following isolated MPFL reconstruction in which patients with a TT-TG greater than or equal to $15 \mathrm{~mm}$ were excluded. In contrast, Caton and Dejour ${ }^{33)}$ reported good to excellent long-term results following TTTs alone, but suggested the need for combined soft tissue procedure to improve outcomes. Wagner et al. ${ }^{34)}$ demonstrated a significant correlation between patellofemoral bony mal-alignment and poor outcome following MPFL reconstruction for chronic patellar instability.

The five articles appraised in this systematic review of the literature represent the best available current evidence on the combined MPFL reconstruction with TTT in the treatment of patellofemoral instability. Combined treatment may offer restoration of normal anatomy, thus potentially adds clinical value to the anatomic approach to MPFL reconstruction. Nevertheless, the current body of evidence does not determine the threshold at which patellofemoral malalignment requires the need for adjunctive treatment as opposed to MPFL reconstruction alone.

A significant limitation in interpreting the current evidence available is the variation in treatment combinations employed to 
treat chronic patellofemoral instability. This systematic review addressed this issue by utilising strict inclusion and exclusion criteria which focused only on combined MPFL and TTT procedures. Table 4 outlines the indications for combined MPFL reconstruction and TTT utilised in the studies included in our review. There was marked heterogeneity in the different articles, which inadvertently mitigates the interpretation of their results. Watanabe et al. ${ }^{30)}$ included second stage MPFL reconstruction ( $n=5$ of 13) for persistent instability following initial tibial tuberosity medialisation. Treatment in the series by Aglietti et al. ${ }^{26)}$ and Cossey and Paterson ${ }^{29)}$ comprised a combination, which also included lateral retinacular release. The latter has been shown to increase lateral patellar instability in selected patients ${ }^{35)}$. Schottle et al. ${ }^{28)}$ selected patients with TT-TG $\geq 15 \mathrm{~mm}$ for the combined treatment.

Collective observations bring into question the efficacy of tibial tuberosity offset correction as an isolated treatment modality in recurrent patellofemoral instability. Accepted normal value for TT-TG had been reported in the range of $10-15 \mathrm{~mm}^{36)}$. Monk et al. ${ }^{37)}$ had previously suggested a measurement of $14.5 \mathrm{~mm}$ as a threshold for unstable patellofemoral alignment. In contrast, Dejour et al. ${ }^{11)}$ used a cutoff point at $20 \mathrm{~mm}$. In the latter series $20 \%$ of asymptomatic knees exceeded this measurement. Equally, Caplan et al. ${ }^{16)}$ demonstrated a lack of difference in TT-TG between stable and unstable knees in the same cohort of patients with recurrent unilateral patellar instability. The use of TT-TG measurement should therefore be viewed with a great deal of caution as a sole indication for surgical correction of patellofemoral malalignment. The current biomechanical, radiological and clinical studies available do not offer sufficient evidence to determine a cut-off point at which patient selection should be decided.

Compounding the issue of patient selection is the paucity of long-term data on the risk and management of failed patellofemoral stabilisation procedures. It is perhaps more plausible to consider the research question of combined versus isolated soft tissue reconstruction by considering the causes of failure of prior surgical treatment. Fithian et al. ${ }^{12)}$ cited a university thesis work by Levigne ${ }^{38)}$ in which $67 \%$ of patients had revision surgery for persistent patellar instability. The latter was linked to a combination of factors, predominantly persistent patellar tilt and persistent patella Alta in $69 \%$ and $45 \%$ of cases respectively ${ }^{38)}$. This work reported that TTM alone was the treatment in $60 \%$ of revised cases compared to soft tissue procedure alone in $30 \%$ of revised cases ${ }^{38)}$. In contrast, Kohn et al. ${ }^{39)}$ reported results of surgical treatment for recurrent patellofemoral instability. In selected cases $45 \%$ occurred following medial plication and lateral release and 12\% following medial plication and TTM. These authors found no difference in revision outcomes when comparing MPFL reconstruction alone with combined MPFL reconstruction and distal realignment ${ }^{39}$. Both series appeared to suggest that the need for revision surgery for persistent patellofemoral instability in their respective selected cases was independent of trochlear morphology ${ }^{38,39)}$. Although widely recognised as an important factor, the role of femoral trochlear shape is beyond the scope of the present review. The natural history of trochlear dysplasia and its relationship to patellofemoral alignment remain contentious topics. In a study looking at the risk of recurrence following distal realignment among a paediatric patient population, remodelling of previously shallow trochlea was identified postoperatively ${ }^{40)}$. In this study Benoit et al. ${ }^{40)}$ analysed the results of skeletally immature patients with a mean age of 10.3 years at a mean follow-up of 13.5 years following distalisation of patellar tendon attachment. Sulcus angles improved by up to a mean of $23.6^{\circ}$ at final followup and were inversely related to age at the time of surgery ${ }^{40)}$.

This systematic review highlighted the lack of clear defining selection criteria and the degree of heterogeneity in patients with patellofemoral instability selected for combined MPFL reconstruction TTT procedure. In addition to study design limitations the outcomes of combined treatment reported were difficult to interpret in light of the varying preoperative status of the cases. There is therefore an emergent need for clearer definition of modalities of the condition and a more optimal clinical and radiological evaluation. Equally, there is a need for clearer definition of intervention groups and selection criteria.

\section{Conclusions}

Results of combined treatment derived from the current available literature remain inconsistent with inadequate evidence to support reduced recurrence of patellar instability. There is limited evidence to suggest that MPFL reconstruction alone might be more appropriate in post-traumatic patellar instability and cases of mild to moderate patellofemoral malalignment. Surgeons should remain prudent in patient selection and must rely on multimodal evaluation of all factors contributing to patellofemoral instability. Consequently, the current body of evidence cannot be used to provide clear recommendations and further research is required to define both patient reported and objective clinical and functional outcome measures. Utilising the latter, priority should be given to randomised controlled trials. Only such robust long-term results can truly support a management algorithm for patellofemoral instability. 


\section{Conflict of Interest}

No potential conflict of interest relevant to this article was reported.

\section{References}

1. Sillanpaa P, Mattila VM, Iivonen T, Visuri T, Pihlajamaki H. Incidence and risk factors of acute traumatic primary patellar dislocation. Med Sci Sports Exerc. 2008;40:606-11.

2. Kim YM, Joo YB. Patellofemoral osteoarthritis. Knee Surg Relat Res. 2012;24:193-200.

3. Sallay PI, Poggi J, Speer KP, Garrett WE. Acute dislocation of the patella: a correlative pathoanatomic study. Am J Sports Med. 1996;24:52-60.

4. Arendt EA, Fithian DC, Cohen E. Current concepts of lateral patella dislocation. Clin Sports Med. 2002;21:499-519.

5. Stephen JM, Kader D, Lumpaopong P, Deehan DJ, Amis AA. Sectioning the medial patellofemoral ligament alters patellofemoral joint kinematics and contact mechanics. J Orthop Res. 2013;31:1423-9.

6. Stephen JM, Lumpaopong P, Deehan DJ, Kader D, Amis AA. The medial patellofemoral ligament: location of femoral attachment and length change patterns resulting from anatomic and nonanatomic attachments. Am J Sports Med. 2012;40:1871-9.

7. Philippot R, Boyer B, Testa R, Farizon F, Moyen B. Study of patellar kinematics after reconstruction of the medial patellofemoral ligament. Clin Biomech (Bristol, Avon). 2012; 27:22-6.

8. Melegari TM, Parks BG, Matthews LS. Patellofemoral contact area and pressure after medial patellofemoral ligament reconstruction. Am J Sports Med. 2008;36:747-52.

9. Camp CL, Krych AJ, Dahm DL, Levy BA, Stuart MJ. Medial patellofemoral ligament repair for recurrent patellar dislocation. Am J Sports Med. 2010;38:2248-54.

10. Elias JJ, Cosgarea AJ. Technical errors during medial patellofemoral ligament reconstruction could overload medial patellofemoral cartilage: a computational analysis. Am J Sports Med. 2006;34:1478-85.

11. Dejour H, Walch G, Nove-Josserand L, Guier C. Factors of patellar instability: an anatomic radiographic study. Knee Surg Sports Traumatol Arthrosc. 1994;2:19-26.

12. Fithian DC, Neyret P, Servien E. Patellar instability: the Lyon experience. Curr Orthop Pract. 2008;19:328-38.

13. Saranathan A, Kirkpatrick MS, Mani S, Smith LG, Cosgarea
AJ, Tan JS, Elias JJ. The effect of tibial tuberosity realignment procedures on the patellofemoral pressure distribution. Knee Surg Sports Traumatol Arthrosc. 2012;20:2054-61.

14. Drexler M, Dwyer T, Marmor M, Reischl N, Attar F, Cameron J. Total knee arthroplasty in patients with excessive external tibial torsion $>45^{\circ}$ and patella instability: surgical technique and follow up. J Arthroplasty. 2013;28:614-9.

15. Lustig S, Servien E, Ait Si Selmi T, Neyret P. Factors affecting reliability of TT-TG measurements before and after medialization: a CT-scan study. Rev Chir Orthop Reparatrice Appar Mot. 2006;92:429-36.

16. Caplan N, Lees D, Newby M, Ewen A, Jackson R, St Clair Gibson A, Kader D. Is tibial tuberosity-trochlear groove distance an appropriate measure for the identification of knees with patellar instability? Knee Surg Sports Traumatol Arthrosc. 2014;22:2377-81.

17. Mani S, Kirkpatrick MS, Saranathan A, Smith LG, Cosgarea AJ, Elias JJ. Tibial tuberosity osteotomy for patellofemoral realignment alters tibiofemoral kinematics. Am J Sports Med. 2011;39:1024-31.

18. Amis AA. Current concepts on anatomy and biomechanics of patellar stability. Sports Med Arthrosc. 2007;15:48-56.

19. Simmons E Jr, Cameron JC. Patella alta and recurrent dislocation of the patella. Clin Orthop Relat Res. 1992;(274):265-9.

20. Ntagiopoulos PG, Sharma B, Bignozzi S, Lopomo N, Colle F, Zaffagnini S, Dejour D. Are the tubular grafts in the femoral tunnel in an anatomical or isometric position in the reconstruction of medial patellofemoral ligament? Int Orthop. 2013;37:1933-41.

21. Wang CH, Ma LF, Zhou JW, Ji G, Wang HY, Wang F, Wang J. Double-bundle anatomical versus single-bundle isometric medial patellofemoral ligament reconstruction for patellar dislocation. Int Orthop. 2013;37:617-24.

22. Liberati A, Altman DG, Tetzlaff J, Mulrow C, Gotzsche PC, Ioannidis JP, Clarke M, Devereaux PJ, Kleijnen J, Moher D. The PRISMA statement for reporting systematic reviews and meta-analyses of studies that evaluate health care interventions: explanation and elaboration. J Clin Epidemiol. 2009; 62:e1-34.

23. Kujala UM, Jaakkola LH, Koskinen SK, Taimela S, Hurme M, Nelimarkka O. Scoring of patellofemoral disorders. Arthroscopy. 1993;9:159-63.

24. Moher D, Hopewell S, Schulz KF, Montori V, Gotzsche PC, Devereaux PJ, Elbourne D, Egger M, Altman DG. CONSORT 2010 explanation and elaboration: updated guidelines for reporting parallel group randomised trials. BMJ. 2010; 
340:c869.

25. Slim K, Nini E, Forestier D, Kwiatkowski F, Panis Y, Chipponi J. Methodological index for non-randomized studies (minors): development and validation of a new instrument. ANZ J Surg. 2003;73:712-6.

26. Aglietti P, Buzzi R, De Biase P, Giron F. Surgical treatment of recurrent dislocation of the patella. Clin Orthop Relat Res. 1994;(308):8-17.

27. Mikashima Y, Kimura M, Kobayashi Y, Asagumo H, Tomatsu T. Medial patellofemoral ligament reconstruction for recurrent patellar instability. Acta Orthop Belg. 2004;70:54550.

28. Schottle PB, Fucentese SF, Romero J. Clinical and radiological outcome of medial patellofemoral ligament reconstruction with a semitendinosus autograft for patella instability. Knee Surg Sports Traumatol Arthrosc. 2005;13:516-21.

29. Cossey AJ, Paterson R. A new technique for reconstructing the medial patellofemoral ligament. Knee. 2005;12:93-8.

30. Watanabe T, Muneta T, Ikeda H, Tateishi T, Sekiya I. Visual analog scale assessment after medial patellofemoral ligament reconstruction: with or without tibial tubercle transfer. J Orthop Sci. 2008;13:32-8.

31. Paxton EW, Fithian DC, Stone ML, Silva P. The reliability and validity of knee-specific and general health instruments in assessing acute patellar dislocation outcomes. Am J Sports Med. 2003;31:487-92.

32. Matthews JJ, Schranz P. Reconstruction of the medial patellofemoral ligament using a longitudinal patellar tunnel technique. Int Orthop. 2010;34:1321-5.

33. Caton JH, Dejour D. Tibial tubercle osteotomy in patello- femoral instability and in patellar height abnormality. Int Orthop. 2010;34:305-9.

34. Wagner D, Pfalzer F, Hingelbaum S, Huth J, Mauch F, Bauer G. The influence of risk factors on clinical outcomes following anatomical medial patellofemoral ligament (MPFL) reconstruction using the gracilis tendon. Knee Surg Sports Traumatol Arthrosc. 2013;21:318-24.

35. Bedi H, Marzo J. The biomechanics of medial patellofemoral ligament repair followed by lateral retinacular release. Am J Sports Med. 2010;38:1462-7.

36. Dandy DJ. Chronic patellofemoral instability. J Bone Joint Surg Br. 1996;78:328-35.

37. Monk AP, Doll HA, Gibbons CL, Ostlere S, Beard DJ, Gill HS, Murray DW. The patho-anatomy of patellofemoral subluxation. J Bone Joint Surg Br. 2011;93:1341-7.

38. Levigne C. Iterative surgery for patellar instability and pain. Analysis of causes of failure and results of re-intervention in 160 cases, in Centre Hospitalier Lyon Sud [thesis]. Villeurbanne: Claude Bernard University Lyon 1; 1988. p146.

39. Kohn LM, Meidinger G, Beitzel K, Banke IJ, Hensler D, Imhoff $A B$, Schottle $P B$. Isolated and combined medial patellofemoral ligament reconstruction in revision surgery for patellofemoral instability: a prospective study. Am J Sports Med. 2013;41:2128-35.

40. Benoit B, Laflamme GY, Laflamme GH, Rouleau D, Delisle J, Morin B. Long-term outcome of surgically-treated habitual patellar dislocation in children with coexistent patella alta: minimum follow-up of 11 years. J Bone Joint Surg Br. 2007; 89:1172-7. 